\title{
BMJ Open Cross-sectional study of the association between healthcare professionals' empathy and burnout and the number of annual primary care visits per patient under their care in Spain
}

Oriol Yuguero, ${ }^{1,2}$ Edward R Melnick, ${ }^{3}$ Josep R. Marsal, ${ }^{4,5}$ Montserrat Esquerda, ${ }^{1,6}$ Jorge Soler-Gonzalez ${ }^{1,2}$

To cite: Yuguero 0 , Melnick ER, Marsal JR., et al. Cross-sectional study of the association between healthcare professionals' empathy and burnout and the number of annual primary care visits per patient under their care in Spain. BMJ Open 2018;8:e020949. doi:10.1136/ bmjopen-2017-020949

- Prepublication history for this paper is available online. To view these files please visit the journal online (http://dx.doi. org/10.1136/bmjopen-2017020949).

Received 5 December 2017 Revised 9 May 2018 Accepted 28 June 2018
Check for updates

(C) Author(s) (or their employer(s)) 2018. Re-use permitted under CC BY-NC. No commercial re-use. See rights and permissions. Published by BMJ.

For numbered affiliations see end of article.

Correspondence to Dr Oriol Yuguero;

Oriol.yuguero@gmail.com

\section{ABSTRACT}

Objective The aim of this study was to evaluate the association between physician and nurse self-reported empathy and burnout and the number of annual primary care visits per patient under their care.

Methods Design: A cross-sectional survey study was conducted from January 2013 to July 2014. Site: The 22 primary care centres of the Lleida Health Region in Spain. Main outcome measures: The Jefferson Scale of Physician Empathy and the Maslach Burnout Inventory were used to measure empathy and burnout, respectively. The number of visits and the number of diagnoses coded per visit were obtained through the Region's electronic health record. Results Two hundred and sixty-seven healthcare professionals (physicians and nurses, $52.6 \%$ participation of the total in the region) with 301657 patients under their care. Healthcare professionals' degree of burnout and empathy was associated with the number of annual visits per patient under their care. Burned out nurses and physicians received fewer visits (4.5vs3.7 in nurses and 18.1 vs18.9 in physicians), whereas more empathic physicians received more visits per patient (19.4vs17.2, $p<0.05)$ and documented more diagnoses per visit (10.2vs9.7, $p=0.001)$. Less burned out and less empathic nurses documented more diagnoses per visit (10.2vs10.0 and 8.2vs9.9, $\mathrm{p}<0.05)$.

Conclusions The number of annual primary care visits per patient that healthcare professionals receive is closely associated with healthcare professionals' empathy and burnout. These results should serve to promote empathic skills and establish organisational changes that promote efficiency in the practice and, in turn, reduce the degree of burnout of healthcare professionals.

\section{BACKGROUND}

The primary care landscape has undergone major changes in recent years. ${ }^{1}$ Administrative burdens, ${ }^{2}$ volume of visits and insufficient resources in times of cutbacks ${ }^{3}$ are increasing burnout among healthcare professionals. Burnout is a syndrome characterised by emotional exhaustion, decreased fulfilment

\section{Strengths and limitations of this study}

- Sample size based on data of more than 300000 patients.

- Use of validated tools to evaluate empathy and burnout.

- The study design does not allow us to establish cause and outcome.

The $52 \%$ response rate could cause selection bias.

and depersonalisation. ${ }^{4}$ Burnout affects healthcare professionals' professional and personal lives leading to physicians reducing their clinical working hours or practice altogether, ${ }^{5}$ thus representing ethical challenges for those responsible for healthcare institutions. ${ }^{6}$ Moreover, burnout has a major impact on the quality of healthcare. ${ }^{78}$ Continuing to deliver high quality primary care with high quality patient relationships requires time. ${ }^{6}$ Time constraints can lead to exhaustion and frustration which are the key elements of burnout.

Front-line physicians with direct patient contact such as those practising primary care, emergency medicine and internal medicine have some of the highest rates of burnout. ${ }^{910}$ In the USA in $2014,55 \%$ of physicians reported symptoms of burnout, ${ }^{11}$ that is, an absolute increase of $10 \%$ in just 3 years. ${ }^{12}$ These findings have prompted individual-level and system-level solutions to combat burnout among healthcare professionals, ${ }^{3} 1314$ especially among young professionals. ${ }^{15}$

Though some individuals may be more prone to burnout, this syndrome is job related and situation specific. ${ }^{16}$ Reducing the level of burnout in healthcare institutions is possible by making it an 
ethical responsibility of institutions to improve professional well-being. ${ }^{17}$ Indeed skills that improve healthcare professionals' empathic capacity have been shown to be associated with lower levels of burnout. ${ }^{181920}$ The theory is that when healthcare professionals understand and communicate patients' situations better, they feel more fulfilled, and they help to humanise care delivery, both of which are fundamental elements in the prevention of burnout. ${ }^{21}$ Since the degree of burnout or professional stress can affect the quality of communication with the patient, this study is particularly relevant given that healthcare professionals are being subjected to increasing clinical workloads and greater time constraints. ${ }^{22}$ Physician stress and burnout are two of the factors that most influence the duration of a primary care visit. ${ }^{23}$

Clinical empathy has been described as the ability to understand others' feelings and thoughts and to communicate such understanding. ${ }^{24}$ Clinical empathy has been shown to be associated with improved communication, patient satisfaction and therapeutic compliance. ${ }^{25} 26$ Empathic physicians reduce patient anxiety, potentially leading to better clinical outcomes. ${ }^{27} 28$

We have evaluated in different studies ${ }^{8}$ how high levels of burnout are linked with little empathy on the part of professionals. ${ }^{18}$ Low empathic capacity hinders communication with patients and in many cases leads to depersonalisation and emotional exhaustion. ${ }^{20}$ These two aspects are fundamental in burnout. In fact, improving healthcare professionals' communication skills has been described as a resource to reduce burnout. ${ }^{21}$

The number of primary care visits per patient is used by the Organization for Economic Co-operation and Development $^{29}$ as one of the measures of healthcare system quality. In 2014, the average number of annual primary care visits per patient in Spain was 7.6 per year per person, above the European average of 7.1 and far higher than the 2.9 annual visits in Sweden.

We wished to prove the effect of professionals with greater burnout on the number of visits they receive but we also thought it would be interesting to see if those professionals with greater empathy received the same number of visits as professionals with less empathy.

Our team believes that empathic professionals solve patients' problems better, and do not need to receive as many visits, and this is related to the cost and quality of healthcare.

The aim of the present study is to evaluate the association between physician and nurse self-reported measures of empathy and burnout and the number of annual primary care visits per patient under their care.

\section{METHODS}

\section{Participants and study design}

A cross-sectional survey study was conducted with volunteer participants. In the Lleida health region, there are 22 primary care centres serving a population of about
366000. All physicians and nurses in the region were contacted by email and asked to complete an anonymous survey that assessed their degree of burnout and empathy.

The study was conducted between January 2013 and July 2014. The survey was administered between May and July 2014.

\section{OUTCOMES}

\section{Burnout and empathy evaluation}

The degree of burnout was measured using the Spanish version of the Maslach Burnout Inventory (MBI), a 22-item scale validated in Spanish. ${ }^{3031}$ This scale measures the three dimensions of burnout: depersonalisation, personal fulfilment and emotional exhaustion. ${ }^{32}$ Empathy was measured using the Spanish version of the Jefferson Scale of Physician Empathy (JSPE), ${ }^{33}$ a validated scale recognised as the gold standard for measuring medical empathy, consisting of 20 items. ${ }^{34}$ Both scales are scored using a 7-point Likert scale, with higher scores indicating higher burnout and greater empathy.

\section{Annual visits per patient}

We analysed the number of visits made by patients to their primary care team (nurse and family physician) between January 2013 and July 2014 (the year in which we collected data from healthcare professionals). The number of visits is the number of contacts with the medical system either with nurses or physicians. The results were divided by 1.4 to obtain the number of visits per calendar year. The number of visits, age and gender of each patient were obtained from the records of the e-CAP electronic health records that are used by all primary care professionals of the Catalan Health Institute. In our healthcare system, the number of visits is automatically recorded as it is mandatory to record the visit in the time table of the professional receiving the visit. It is important to note that the number of visits by each patient is different from the volume of visits for which a healthcare professional was responsible during that year. Given the varying roles and responsibilities of physicians and nurses within a single care team, we calculated separate values for this outcome for physicians and nurses.

\section{Number of diagnoses coded per visit}

We collected the number of diagnoses that the participant healthcare professionals documented for each visit. The number and type of diagnoses were used to classify the severity and complexity of the visit. The diagnoses included in our analysis were diabetes, heart failure, ischaemic heart disease, stroke, dyslipidaemia, hypertension, anaemia, joint fibrillation, chronic renal failure, apnoea, anxiety, depression, metabolic syndrome. So, for a hypothetical patient with no diagnoses, the number of diagnoses would be zero.

We defined the diagnoses (ie, diabetes, heart failure, ischaemic heart disease, etc) from the electronic records of the medical history (e-CAP). All the diagnoses were 
recorded from the practitioners using the International Classification of Diseases 10th Edition dictionary. For each diagnosis, a binary variable was defined, indicating presence or absence, and the sum of all of them.

\section{Participant characteristics}

The following sociodemographic data were collected for the practitioners: age, gender, professional category (physician or nurse) and practice setting (urban or rural).

\section{Data analysis}

Standard descriptive summary statistics were used to characterise the MBI and JSPE scores. The reliability of the instruments was tested using Cronbach's $\alpha$.

The $\chi^{2}$ and Kolmogorov-Smirnov tests were used to evaluate the distribution of these scores. To analyse the association between the sociodemographic variables and the results of the JSPE, MBI and number of visits, the results were grouped into three categories (low, medium and high) using previously described value ranges and categories. ${ }^{12}$ All results were to be presented with a $95 \%$ CI. Results of association were compared using the $\chi^{2}$ test. The results were disaggregated according to age, gender, professional category and practice setting. For data analysis, means, percentages and SD were calculated using SPSS V.15.0 (IBM 2006) software.

\section{Confidentiality consideration}

The data were kept confidential and anonymous in accordance with the Spanish Data Protection Law 15/1999.
All data were coded and accessible only to the primary care information system technicians who cross-referenced the data. All data were deidentified before being made available to the investigators.

\section{Patient and public involvement}

No patients or public were involved in the study.

\section{RESULTS}

Of the 267 healthcare professionals who participated in the study (response rate of $52.6 \%$ of all practitioners in the region), 131 (49\%) were nurses, $136(51 \%)$ were physicians, $209(78.3 \%)$ were women and 156 (58.4\%) worked in rural areas. This sample was representative of the whole population of healthcare practitioners in the region according to the Ministry of Health of Catalonia. We have included data on sociodemographic variables in table 1. No significant differences were detected between burnout and gender or professional role. Medical professionals practising in rural areas reported a lower degree of empathy $(\mathrm{p}<0.05)$ but no significant differences in burnout. High empathy was associated with low burnout in both nurses and physicians $(\mathrm{p}<0.05)$, Cronbach's $\alpha$ was 0.733 for the MBI and 0.748 for the JSPE, which shows adequate reliability of the scales used.

\section{Annual visits per patient}

We analysed the annual number of visits per patient from the 301657 patients under the care of the 267 participating

\begin{tabular}{|c|c|c|c|c|c|c|c|}
\hline & \multicolumn{2}{|c|}{ Urban $(n=111)$} & \multicolumn{2}{|c|}{ Rural $(n=156)$} & \multicolumn{2}{|c|}{ Total $(n=267)$} & \multirow[b]{2}{*}{ P values } \\
\hline & $\mathbf{N}$ & n (\%) & $\mathbf{N}$ & n (\%) & $\mathbf{N}$ & n (\%) & \\
\hline Age (years) & 111 & & 156 & & 267 & & 0.915 \\
\hline $31-40$ & & $26(23.4)$ & & $34(21.8)$ & & $60(22.5)$ & \\
\hline $41-50$ & & $40(36)$ & & $55(35.3)$ & & 95 (35.6) & \\
\hline$>50$ & & 45 (40.5) & & 67 (42.9) & & $112(41.9)$ & \\
\hline Professional role & 111 & & 156 & & 267 & & 0.405 \\
\hline Nurse & & $50(45)$ & & 81 (51.9) & & $131(49.1)$ & \\
\hline Physician & & $61(55)$ & & $75(48.1)$ & & 136 (50.9) & \\
\hline Gender & 111 & & 156 & & 267 & & 0.349 \\
\hline Men & & 21 (18.9) & & 37 (23.7) & & $58(21.7)$ & \\
\hline Women & & $90(81.1)$ & & 119 (76.3) & & 209 (78.3) & \\
\hline Empathy (JSPE) & 111 & & 156 & & 267 & & 0.018 \\
\hline Low & & 27 (24.3) & & 62 (39.7) & & 89 (33.3) & \\
\hline Medium & & $38(34.2)$ & & $50(32.1)$ & & $88(33)$ & \\
\hline High & & $46(41.4)$ & & $44(28.2)$ & & $90(33.7)$ & \\
\hline Burnout & 111 & & 156 & & 267 & & 0.774 \\
\hline Low & & $63(56.8)$ & & $94(60.3)$ & & 157 (58.8) & \\
\hline Medium & & $43(38.7)$ & & $57(36.5)$ & & $100(37.5)$ & \\
\hline High & & $5(4.5)$ & & $5(3.2)$ & & $10(3.7)$ & \\
\hline
\end{tabular}

JSPE, Jefferson Scale of Physician Empathy. 
Table 2 Characteristics of patients according to empathy and burnout of nursing staff

\begin{tabular}{|c|c|c|c|c|c|}
\hline \multirow[b]{2}{*}{ Empathy } & Low $(n=52173)$ & Medium ( $n=51298$ ) & High $(n=49354)$ & Total $(n=152825)$ & \multirow[b]{2}{*}{$P$ values } \\
\hline & Mean (SD) & Mean (SD) & Mean (SD) & Mean (SD) & \\
\hline Women patients & $25851(49.5 \%)$ & 25290 (49.3\%) & 24452 (49.5\%) & 75593 (49.5\%) & 0.977 \\
\hline Age (years) & $48.1(19.1)$ & $48.4(19.2)$ & $48.5(19.4)$ & $48.3(19.2)$ & 0.014 \\
\hline Visits 2014 & $4.5(6.9)$ & $4.4(6.6)$ & $4.4(6.6)$ & $4.5(6.7)$ & 0.065 \\
\hline Visits 2012 & $18.9(23.7)$ & $18.6(23.2)$ & $18.6(23)$ & $18.7(23.3)$ & 0.075 \\
\hline \multirow[t]{3}{*}{ Number of diagnoses } & $10.2(8.5)$ & $9.7(8.3)$ & $10(8.3)$ & $10(8.4)$ & 0.001 \\
\hline & High $(n=1496)$ & Medium $(n=54441)$ & Low $(n=968888)$ & Total $(n=152825)$ & \\
\hline & Mean (SD) & Mean (SD) & Mean (SD) & Mean (SD) & $P$ values \\
\hline Women patients & 788 (52.7\%) & 27167 (49.9\%) & 47638 (49.2\%) & 75593 (49.5\%) & 0.001 \\
\hline Age (years) & $48.6(18.8)$ & $47.6(18.8)$ & 48.7 (19.5) & $48.3(19.2)$ & 0.001 \\
\hline Visits 2014 & $3.7(5.3)$ & $4.3(6.4)$ & $4.5(6.8)$ & $4.5(6.7)$ & 0.001 \\
\hline Visits 2012 & $16.1(19.7)$ & $18.1(22.1)$ & $19.1(24)$ & 18.7 (23.3) & 0.001 \\
\hline Number of diagnoses & $8.4(6.7)$ & $10.2(8.4)$ & $9.9(8.4)$ & $10(8.4)$ & 0.001 \\
\hline
\end{tabular}

healthcare professionals. Nurses with higher burnout received fewer annual visits per patient (4.5 visits vs 3.7 in the most burned out, $\mathrm{p}=0.001$, table 2 ). There was no significant difference in the number of annual visits per patient based on nurses' degree of empathy. The most burned out physicians received fewer annual visits per patient (18.1 vs $18.9, \mathrm{p}=0.002$, table 3 ). Physicians with less empathy received a higher number of visits by their patients (19.4 vs $17.2, \mathrm{p}=0.001)$.

\section{Number of diagnoses coded per visit}

Less burned out nurses (8.4 vs 9.9, $\mathrm{p}<0.05)$ and less empathic nurses (10 vs $10.2, \mathrm{p}<0.05)$ documented more diagnoses per visit, whereas physicians with medium range empathy documented the most diagnoses (10.2 vs $9.7, \mathrm{p}=0.001$ ). In addition, physicians with the highest degree of burnout were the ones who documented the most diagnoses per visit $(10.2$ vs $10, \mathrm{p}<0.05)$.

\section{DISCUSSION}

In this cross-sectional survey study, we found a significant association between primary care healthcare professionals' burnout and empathy and the annual number of visits per patient under their care. This large, highly representative sample is the first (to our knowledge) to analyse this association and is strengthened by the inclusion of both physicians and nurses. Few similar studies make it difficult to compare our results to the existing literature.

The healthcare professionals' degree of burnout and level of empathy were associated with the annual number of visits per patient under their care. The most empathic and least burned out physicians received fewer visits. We hypothesise that this relationship could be due to the fact that these physicians can better solve their patients' problems with fewer visits. We were unable to compare these

Table 3 Characteristics of patients based on empathy and burnout of physicians

\begin{tabular}{|c|c|c|c|c|c|}
\hline & Low $(n=42138)$ & Medium $(n=45070)$ & High $(n=61624)$ & Total $(n=148832)$ & \\
\hline Empathy & Mean (SD) & Mean (SD) & Mean (SD) & Mean (SD) & $P$ values \\
\hline Women patients & $20793(49.3 \%)$ & $22246(49.4 \%)$ & 30765 (49.9\%) & 73804 (49.6\%) & 0.052 \\
\hline Age (years) & $48.9(19.3)$ & $48.9(19.4)$ & $47.9(19)$ & 48.5 (19.2) & 0.001 \\
\hline Visits 2014 & $4.6(6.7)$ & $4.5(6.7)$ & $4.1(6.3)$ & $4.4(6.5)$ & 0.001 \\
\hline Visits 2012 & $19.4(23.5)$ & $18.9(23.8)$ & $17.2(21.7)$ & $18.3(22.9)$ & 0.001 \\
\hline \multirow[t]{2}{*}{ Number of diagnoses } & $9.7(7.8)$ & $10.2(8.6)$ & $9.7(8.3)$ & $9.8(8.3)$ & 0.001 \\
\hline & High $(n=9660)$ & Medium $(n=57742)$ & Low $(n=81430)$ & Total $(n=148832)$ & \\
\hline Burnout & Mean (SD) & Mean (SD) & Mean (SD) & Mean (SD) & $P$ values \\
\hline Women patients & $4676(48.4 \%)$ & $28798(49.9 \%)$ & $40330(49.5 \%)$ & $73804(49.6 \%)$ & 0.589 \\
\hline Age (years) & $47.9(18.7)$ & $48.6(19.3)$ & $48.5(19.1)$ & 48.5 (19.2) & 0.003 \\
\hline Visits 2014 & $4.5(6.6)$ & $4.4(6.5)$ & $4.4(6.5)$ & $4.4(6.5)$ & 0.069 \\
\hline Visits 2012 & $18.9(22.9)$ & $18.4(23.1)$ & $18.1(22.8)$ & $18.3(22.9)$ & 0.002 \\
\hline Number of diagnoses & $10.2(8.8)$ & $9.6(7.9)$ & $10(8.5)$ & $9.8(8.3)$ & 0.001 \\
\hline
\end{tabular}


results with other similar ones, since to date the literature ${ }^{10}$ has only related the severity of consultation with the duration of the consultation, not with the number of encounters between physician and patient.

However, among nurses, the associations we found were different. Nurses with less burnout received a greater number of consultations. We should consider that tasks performed by nurses were generally associated with cures, healthcare promotion and case management. ${ }^{35} \mathrm{We}$ hypothesise that the nature of nurses' roles and responsibilities within the care team could influence this relationship, that is, patients may perceive that they can consult the nurse more in a single visit without encountering resistance. If so, less burned out nurses may not have minded receiving more visits by the same patient, to follow up and monitor the evolution of the patient's problems. ${ }^{36}{ }^{37}$ Also in the field of nursing, we suspect this greater autonomy of visits and case management may be related to greater professional satisfaction. ${ }^{38}$ Likewise, the professional situation also has an association in the documentation of the patients' diagnoses.

In reference to the number of diagnoses coded per visit, we believe that the results we have obtained reflect an association with the professional situation. As for empathy, both less empathic nursing staff and physicians document more diagnoses per visit. We hypothesise that professionals with better communication (and empathy) skills spend more time with the patient and less time documenting diagnoses. It should be noted that the recording of diagnoses in the computer program is important for two main reasons. On the one hand, these diagnoses can serve as a rapid reference for other healthcare professionals caring for the same patient. On the other hand, the patient's clinical complexity is determined by the coded diagnoses, thus qualifying for certain clinical programmes (ie, inclusion in domiciliary healthcare programmes or palliative care) may depend on correct coding. For these reasons, we believe that healthcare professionals with medium levels of empathy are the ones who focus on patient care at the interview but also understand the importance of the health records.

However, it is striking that in the case of physicians, the most burned out are the ones who record the most visits. This finding has been described previously, ${ }^{39}$ that is, that burned out healthcare professionals are more likely to dehumanise their patients and focus more on the iPatient than the actual human being in front of them. Similarly in Spain, documentation of more diagnoses increases financial incentives linked to quality indicators. ${ }^{8}$

We acknowledge several limitations to our study including the use of self-reported outcomes which, though validated and widely used, could lead to a reporting bias. Furthermore, the 52\% response rate could cause selection bias. In our region, a large number of healthcare professionals work in rural areas where access to family physicians and nurses (given the great geographical dispersion $)^{40}$ may be more difficult than in urban areas. There is also another bias, the number of hours the nurse or physician is working. This information could be important to evaluate the effect on empathy/burnout or number of visits. In addition, the majority of healthcare professionals are over 50 years of age. The study design does not allow us to establish cause and outcome. We have chosen this interpretation but we must assume that interpretations could be made in other directions. Finally, we think it would be positive to develop a multivariate analysis, to evaluate different factors affecting empathy and burnout. However, our database was not done with that objective, and it would be a good option for further research.

The work relating empathy with burnout in our health region is a first in our country and has managed to verify a reality that has been widely described in other countries, that is, the association that exists between the degree of empathy and burnout of professionals and the number of visits they receive.

We also consider an interesting line of further investigation would be to perform a qualitative study in order to detect differences between doctors and nurses, and to analyse the relationship between teamwork and its influence on burnout.

Based on the results, we believe that healthcare institutions should continue to promote communication skills and other work relationship initiatives that reduce burnout among healthcare professionals. This would surely help to improve healthcare and affect quality indicators. An interesting line would be the performance of a qualitative study to detect differences between doctors and nurses, and thus to be able to develop the concept of the grouping of empathy.

In conclusion, we believe that future research should focus on which communication skills and work situations can improve the quality of care. Promoting such skills could lead to an improvement not only in the clinical quality of care but also in the working environment. Burnout levels have been linked with work effort. One of the most important implications of our study is to quantify the effect of healthcare professionals' burnout on patient care. ${ }^{41}$ Healthcare policy-makers should be aware of the different measures that can reduce professional burnout (promoting professional engagement, team building, flexible work schedule,...). Perhaps our findings should encourage introspection on the alignment of financial incentives based on communication and empathy rather than on traditional quality indicators like the number of diagnoses entered in the electronic health record. We believe that the results of our study may prove interesting for healthcare organisation leaders to encourage programmes that promote empathic skills and to establish strategies that reduce the degree of burnout of healthcare professionals to improve the quality of patient care.

\section{Author affiliations}

${ }^{1}$ Faculty of Medicine, University of Lleida, Lleida, Spain

${ }^{2}$ Biomedical Research Institute of Lleida, IRBLLEIDA, Lleida, Spain 
${ }^{3}$ Department of Emergency Medicine, Yale School of Medicine, New Haven, Connecticut, USA

${ }^{4}$ Research Support Unit, Primary Care Research Institute (IDIAP), Barcelona, Spain ${ }^{5}$ Epidemiology Unit, Cardiovascular Department, Vall d'Hebron University Hospital, Barcelona, Spain

${ }^{6}$ Direction, Borja Bioethics Institute, Barcelona, Spain

Acknowledgements The English translation of this article was rendered with the support of the Languages Institute of the University of Lleida. We want to thank to all the professionals who participated in the study.

Contributors OY designed the study and wrote the main part of the paper. ERM collaborated in the design of the paper and in its revision. JRM performed the statistical analysis. ME collaborated in the collection of data and in the introductory research. JS-G reviewed the manuscript and collaborated in the revision of the entire process.

Funding This study was funded by Universitat de Lleida (translation of the paper). Competing interests None declared.

Patient consent Not required.

Ethics approval Clinical Research Ethics Committee of the Jordi Gol Institute for Primary Care Research (IDIAP).

Provenance and peer review Not commissioned; externally peer reviewed.

Data sharing statement All the data are included in the article.

Open access This is an open access article distributed in accordance with the Creative Commons Attribution Non Commercial (CC BY-NC 4.0) license, which permits others to distribute, remix, adapt, build upon this work non-commercially, and license their derivative works on different terms, provided the original work is properly cited, appropriate credit is given, any changes made indicated, and the use is non-commercial. See: http://creativecommons.org/licenses/by-nc/4.0/.

\section{REFERENCES}

1. Casado V. Construyendo la atención primaria española en una Europa cambiante. Aten Primaria 2016;48:71-2.

2. Shanafelt TD, Dyrbye LN, Sinsky C, et al. Relationship between clerical burden and characteristics of the electronic environment with physician burnout and professional satisfaction. Mayo Clin Proc 2016;91:836-48.

3. Simó J, Gérvas J. Gasto sanitario en atención primaria en España: insuficiente para ofrecer servicios atrayentes para pacientes y profesionales. Informe SESPAS 2012. Gac Sanit 2012;26:36-40.

4. Maslach C. Burnout: the cost of caring: Englewood Cliffs. N.J. Prentice Hall, 1982.

5. Olson KD. Physician burnout-a leading indicator of health system performance? Mayo Clin Proc 2017;92:1608-11.

6. Shanafelt TD, Noseworthy JH. Executive leadership and physician well-being: nine organizational strategies to promote engagement and reduce burnout. Mayo Clin Proc 2017;92:129-46.

7. Dewa CS, Loong D, Bonato S, et al. The relationship between physician burnout and quality of healthcare in terms of safety and acceptability: a systematic review. BMJ Open 2017;7:e015141.

8. Yuguero O, Marsal JR, Buti M, et al. Descriptive study of association between quality of care and empathy and burnout in primary care. BMC Med Ethics 2017;18:54.

9. Shanafelt TD, Boone S, Tan L, et al. Burnout and satisfaction with work-life balance among US physicians relative to the general US population. Arch Intern Med 2012;172:1377-85.

10. Dyrbye L, Shanafelt T, Sinsky C, et al. Burnout among health care professionals: a call to explore and address this underrecognized threat to safe, high-quality care. NAM Perspectives. Discussion Paper. Washington, DC: National Academy of Medicine, 2017.

11. Medscape LifeStyle Report 2016. 2016. http://www.medscape.com/ features/slideshow/lifestyle/2016/public/overview\#page $=5$

12. Shanafelt TD, Hasan O, Dyrbye LN, et al. Changes in burnout and satisfaction with work-life balance in physicians and the general us working population between 2011 and 2014. Mayo Clin Proc 2015;90:1600-13.

13. Burgess DJ, Beach MC, Saha S. Mindfulness practice: a promising approach to reducing the effects of clinician implicit bias on patients. Patient Educ Couns 2017;100:372-6.
14. Bowman MA, Seehusen DA, Victoria Neale A. Interventions must be realistic to be useful and completed in family medicine. J Am Board Fam Med 2018;31:1-4.

15. Hansen A, Peterson LE, Fang B, et al. Burnout in young family physicians: variation across states. J Am Board Fam Med 2018;31:7-8.

16. Melnick ER, Powsner SM, Shanafelt TD. In reply-defining physician burnout, and differentiating between burnout and depression. Mayo Clin Proc 2017;92:1456-8.

17. Shanafelt T, Goh J, Sinsky C. The business case for investing in physician well-being. JAMA Intern Med 2017;177:1826-32.

18. Yuguero $O$, Forné $C$, Esquerda $M$, et al. Empathy and burnout of emergency professionals of a health region: a cross-sectional study. Medicine 2017;96:e8030.

19. Gleichgerrcht E, Decety J. Empathy in clinical practice: how individual dispositions, gender, and experience moderate empathic concern, burnout, and emotional distress in physicians. PLoS One 2013;8:e61526.

20. Yuguero O, Ramon Marsal J, Esquerda M, et al. Association between low empathy and high burnout among primary care physicians and nurses in Lleida, Spain. Eur J Gen Pract 2017;23:1-7.

21. Melnick ER, Powsner SM. Empathy in the time of burnout. Mayo Clin Proc 2016;91:1678-9.

22. Brazeau CM, Schroeder R, Rovi S, et al. Relationships between medical student burnout, empathy, and professionalism climate. Acad Med 2010;85:S33-S36.

23. Orton PK, Pereira Gray D. Factors influencing consultation length in general/family practice. Fam Pract 2016;33:529-34.

24. Hojat M, Gonnella JS, Nasca TJ, et al. Physician empathy: definition, components, measurement, and relationship to gender and specialty. Am J Psychiatry 2002;159:1563-9.

25. Zachariae R, Pedersen CG, Jensen AB, et al. Association of perceived physician communication style with patient satisfaction, distress, cancer-related self-efficacy, and perceived control over the disease. Br J Cancer 2003;88:658-65.

26. Kelley JM, Kraft-Todd G, Schapira L, et al. The influence of the patient-clinician relationship on healthcare outcomes: a systematic review and meta-analysis of randomized controlled trials. PLoS One 2014;9:e94207.

27. Derksen F, Bensing J, Lagro-Janssen A. Effectiveness of empathy in general practice: a systematic review. Br J Gen Pract 2013;63:76-84.

28. Hojat M, Louis DZ, Markham FW, et al. Physicians' empathy and clinical outcomes for diabetic patients. Acad Med 2011;86:359-64.

29. OCDE. Health Statisticis 2016. Disponible en. 2016. http://stats. oecd.org/Index.aspx?DataSetCode=HEALTH_PROC

30. Moreno-Jiménez B, Carvajal R, Escobar R. The evaluation of professional Burnout. Factorialization of the MBI-GS. A preliminary analysis. Ansiedad y Estrés 2001;7:69-78.

31. Yuguero Torres O, Esquerda Aresté M, Marsal Mora JR, et al. Association between Sick Leave Prescribing Practices and Physician Burnout and Empathy. PLoS One 2015;10:e0133379.

32. Álvarez E, Fernández L. El síndrome de burnout o el desgaste profesional. [The burnout syndrome or professional burnout. Rev Asoc Esp Neuropsiq 2001;21:257-65.

33. Alcorta-GarzaA G-GJF, Tavitas-Herrera S. Validación de la escala de empatía médica de Jefferson en estudiantes de medicina mexicanos [Validation of Jefferson scale of empathy among Mexican medical students]. Salud Mental 2005;28:57-63.

34. Hojat M. The Jefferson scale of physician empathy: further psychometric data and differences by gender and speciality at item level. Acad Med 2002;7:S58-60.

35. Brugués Brugués A, Peris Grao A, Pavón Rodríguez F, et al. [Evaluation of nurse demand management in primary care]. Aten Primaria 2016;48:159-65.

36. Dempsey C, Reilly BA. Nurse engagement: what are the contributing factors for success? Online J Issues Nurs 2016;21:2.

37. Navarro-González D, Ayechu-Díaz A, Huarte-Labiano I. [Prevalence of burnout syndrome and its associated factors in Primary Care staff]. Semergen 2015:41:191-8.

38. Lorber M, Skela Savič B, Skela B. Job satisfaction of nurses and identifying factors of job satisfaction in Slovenian Hospitals. Croat Med J 2012;53:263-70.

39. Verghese A. Culture shock--patient as icon, icon as patient. $N$ Engl J Med 2008;359:2748-51.

40. Arroyo Al, Guerrero O, Barneto A, et al. "Luces y sombras de la medicina rural: a propósito de la docencia". Aten Primaria 2007;39:219-20.

41. Shanafelt TD, Mungo M, Schmitgen J, et al. Longitudinal study evaluating the association between physician burnout and changes in professional work effort. Mayo Clin Proc 2016;91:422-31. 